\title{
ONTOLOGY AND THE MATHEMATIZATION OF THE SCIENTIFIC ENTERPRISE
}

\author{
Décio Krause Jonas R. B. Arenhart \\ Newton C. A. da Costa \\ Department of Philosophy Federal University of Santa Catarina
}

\section{Introduction}

In this expository paper we shall consider one foundational aspect of the philosophical discussion of scientific theories. Our main purpose is to throw some light in the role played by the underlying logic and mathematics of a theory in the ontological counterpart of the scientific enterprise; we shall investigate how those usually unacknowledged parts of a scientific theory contribute to substantiate an ontology associated with a theory.

By considering that today we have distinct and non-equivalent logical and mathematical systems (for instance, different and non-equivalent set theories), an immediate question poses itself: are we allowed to choose among some of the available system(s) to ground our scientific credos or, alternatively, are we necessarily committed to a certain (a priori and necessary) system of logic and mathematics? Furthermore, how does the use of alternative underlying logics influence in the possible ontologies of a theory? We shall defend a version of the view that alternative systems of logic may be used profitably to formulate and study the ontology of a theory. However, despite the plurality of those systems, it is not the case that anything goes; as we mentioned, the theory itself puts constraints on the possible ontologies that may reasonably be called upon for the interpretation of a theory.

Our main contention in this paper is that there is a mutual influence between the logic and the ontology of a scientific theory. On the one hand, logic encapsulates the most general categories of the theory and describes how these categories work in general, providing for their principles. Thus, on one 
side logic imposes from the bottom some restrictions on the possible ontologies allowed for a theory; on the other side, scientific theories impose from the top constrains on the objects to be dealt with, helping us also to shape the (supposed) most adequate underlying logic (i.e. a logic 'reflecting' the general features of those objects). This is a two way street, and we shall elaborate on its possible paths and where they may lead to in the specific case of non-relativistic quantum mechanics. Some hints involving quantum field theory are also mentioned en passant, but just to exemplify some situation.

The overall plan of this paper is as follows. We begin by presenting in the next section a general scheme that purports to describe in a rough way the relation of a theory with reality. It shall be useful to help us make our points on the relation of the underlying logic and a theory clearer. In section 3 we discuss what we call 'classical ontology' (a view grounded on classical logic, which has intersections with both standard mathematics and classical mechanics) and its relation to classical logic. In section 4 we discuss an ontology inspired by quantum mechanics, termed 'quantum ontology' and analyze how it may contribute to the suggestion that an alternative system of logic could accommodate more profitably those features. We continue by discussing the other side of the coin, namely, how (and if) logic might influence ontology by imposing general features to objects, and conclude by reinforcing the claim that logic is sensitive to the demands of empirical theories, so that it needs not to be totally a priori.

\section{A general scheme}

We start by presenting in this section a general scheme that may be useful for the philosophical study of ontological aspects of scientific theories. We are not claiming neither that this is the sole possible scheme nor that all scientific theories should be understood according to it. Its main purpose is to help us to illustrate in an idealized way the different steps in the elaboration of a scientific theory and how ontological discussions may benefit from a closer look to foundations in philosophy of science. Important to note that we are not claiming that scientists make use of schema like this one, for apparently there are no rules for the elaboration of a theory. Our scheme serves merely to the purpose of enlightening some steps which are usually subsumed in the activities proper of science. Let us consider the following sequence of steps:

$$
R \Rightarrow E R \Rightarrow I T \Rightarrow T \Rightarrow A
$$


Some explanations are in order. We may may assume a form of metaphysical realism according to which something exists independently of us, even if it cannot be described in full even by our best theories (put up to now). Let us use the letter $R$ for such a 'reality', although it could perhaps remain forever 'veiled' to us [7, Chap.9]. Of course we could adopt a different strategy and suppose that there is no such a reality but just our phenomenological insights, which we call 'Empirical Reality', or 'Phenomenological Sense-Experience Reality', $E R$ for short. Thus, in a non-realistic view, we could assume that we should start with $E R$, so ignoring $R$; our scheme can also be understood this way, so it is neutral as to the existence and possibility of theoretical knowledge of an independent reality, for it is also coherent with the view that there is nothing behind the appearances. Obviously, we here acknowledge that reality, as it is, is partially inaccessible to our sense experience. ${ }^{1}$ All we get from (a supposed existing) $R$ is sense-data, derived from experiences, sensations, reflection, intuitions, learning, insights, whatever you will. In this sense, it is allowed that different experiences, cultural background, biological (animal) skills, etc., may interfere in the way we further elaborate our sense-experiences. It is from this $E R$ that we elaborate our conceptual schemæ that gives us our scientific theories, usually in an informal way to start with, that is, by presenting a non axiomatized or formalized account of at least a parcel of $E R .^{2}$

Another remark concerning $R$ is in order. One could suppose that we are referring to $R$ in Husserl's terms, as das Reale, a term which refers to the spatiotemporal world as conceived by physics (see [13, p.23, fn.1]). But, as we shall see below, we can say that there is not just one ontology conceived by physics, for the domains of application may appear different depending on the physical theory we are considering and, furthermore, depending on the part of the world we are investigating, different theories seem to be required, since there is not yet a unified general schema that applies to all scales. ${ }^{3}$ Here, $R$ stands for a reality that is independent of us. The world, as conceived by physics, enters in our $I T$ s, which concentrate our 'representations', and their format depend on the way we formulate our theories.

1 We say, following P. Kosso, "at least partially" inaccessible. Kosso defends what he calls "realistic realism", acknowledging that (according to realists) we can know something about the world, but not everything can be known. See his [14], specially chapter 8 .

2 But of course nothing prevents that an axiomatized theory may be proposed from the start. We are speaking about the most general and more common way of doing science. Here, 'informal' means 'non axiomatized'.

3 In fact, there are different physical theories on the market, and no unification is seen up to now. 
The sense-data collected in ER are elaborated in terms of more abstract concepts. The scientific activity is a conceptual activity, and we use our skills to link them in order to get a coherent whole which will serve to provide us the way for making explanations, predictions and retrodictions, providing our understanding of a situation. Thus we may speak of a theory. But, since we wish to use the word 'theory' tout court to mean an axiomatized or a formalized axiomatization of a field of knowledge, we prefer to call what we have achieved an informal theory, IT for short. An IT can be viewed as a 'modeling' concepts we have in form of an articulated framework that enables us to get other results (supposedly) concerning a certain parcel of reality we are interested in. Of course there are (in principle) infinitely many possibilities for developing an $I T$, depending on the scientist's preferences and on other factors. Furthermore, nothing excludes that a scientist presents 'directly' a theory properly speaking, that is, an axiomatized or a formalized theory of the domain under investigation (the distinction between axiomatization and formalization shall be explained soon). Aristotle's physics (see [27]), Galileo's mechanics [10], Darwin's evolution theory, Dewey's educational theory [6], Heisenberg's matrix mechanics, Schrödinger's wave mechanics, Einstein's general relativity, are typical examples of $I T$ s (see [23]).

Thus, the Informal Theories (IT s) we find in the standard literature are usually theoretical (mathematical) elaborations that may be achieved in various distinct ways. Just remember that Heisenberg and Schrödinger have advanced alternative informal theories to the same subject, just as there are different evolution theories that can be found in the literature (Wallace, Darwin, Lamarck, etc.). Similarly, we should acknowledge that classical mechanics has different formulations, such as those of Newton, Lagrange, Hamilton, and Hamilton- Jacobi, at least. When constructing such a scientific theory, mainly in the field of physics, we make use of mathematical concepts such as derivatives, differential equations, statistics, and abstract spaces of several kinds. Even in biology we can suppose that some mathematics is used, say in techniques taken from statistics, combinatorial analysis, lattice theory, and so on. In economy, presently much of differential and integral calculus has been used in some contexts and, generalizing, we can extend this hypothesis to scientific $I T$-theories in general.

In general, IT s are what scientists aim at, and to most of their purposes they are enough. But the philosopher and those occupied with foundations may go further, looking for the logical structure of the achieved IT. Here a meta-level discussion takes place, for we are no longer discussing the applicability of the scientific constructs to reality, but we are rather looking to the constructs themselves. This becomes clear when we try to go from the 
informal theories $I T$ to their axiomatic versions, which we call theories (tout court).

But, why to axiomatize? It is well known that the rise of the movement called the Arithmetization of Analysis started in the XIXth century from the dissatisfaction of mathematicians with the basic informal concepts they used, such as those of function and real number, which were not rigorous as required for foundational purposes. In physics, Newton's mechanics generalizes more or less axiomatically the informal developments advanced by Galileo, Kepler and others. Maxwell's electromagnetic theory, with some parsimony, can be seen as an axiomatic framework, once we consider the possibility of presenting an adequate language and taking his famous equations in the place of axioms. In the XXth century, much was done in the axiomatizations in biology and in other areas as well (see [22], [31]). By the way, this is the core of Hilbert's 6th problem of his Mathematical Problems, posed in 1900 (see [1]). With the rise of abstract mathematical structures by the end of the XIXth century, and with the parallel development of logic, axiomatization was seen as the method par excellence of mathematical theories, and the XXth century has shown that it can be useful for other areas as well.

What results from axiomatization? Here axiomatization can be taken in Hilbert's sense, either as a material axiomatization or as a formal axiomatization. Material axiomatics (by hypothesis) do not lose the intuitive and intended meaning of the informal theory. Peano's (first-order) Arithmetics is supposed to deal with arithmetics, with the natural numbers we have from our intuitive mathematics (the existence of non-standard models will be recalled soon). Indeed, we are axiomatizing a certain informal theory and believing that the axiomatic version fits the previous (informal) framework. For instance, there are several axiomatizations of the theory of evolution, and all of them were proposed to axiomatize evolution as firstly understood by Wallace and Darwin, although there are also axiomatizations of more recent accounts, say incorporating genetics (see [19] and the references therein; in [22] there is a wider discussion on the axiomatization of biology).

To be more precise, let us assume, as Suppes has famously proposed, ${ }^{4}$ that to axiomatize a theory (an informal theory) is to present a predicate in the language of set theory [31]. (Here we are being a little more rigorous - a dis-

${ }^{4}$ Of course this procedure cannot be applied in general without due qualification; for instance, it doesn't not serve to intuitionistic mathematics. Furthermore, if we strictly follow Suppes, we become committed with classical logic and can consider only scientific theories based on such a logic. The reader may note that we are here just exemplifying that in axiomatizing a informal field, we create something that cannot be said without reservations it fits correctly that field. 
cussion about Suppes' method and what was later called 'Suppes predicates' can be found in [16], [15]). In presenting such a predicate, we are evolving from an informal theory to an axiomatized version, which then becomes abstract, gaining a certain autonomy from its first intended domain of application. Speaking in terms of Suppes' predicates, a predicate may have infinitely many mathematical structures as models, namely, the structures satisfying the predicate. For instance, a predicate (set of axioms) for first-order Peano's arithmetics will have not only the standard model as a model, but many other non-standard models as well.

A simple example illustrates the idea. Assuming classical logic and a standard set theory, think of a semi-group, which is an ordered pair composed of a non-empty set $S$ and a binary operation on $S$ that is associative. The predicate may be written in a simplified way (in the language of set theory) as follows:

$$
\begin{aligned}
\mathrm{P}(X) \Leftrightarrow \exists S \exists \star(X=\langle S, \star\rangle \wedge S \neq \emptyset \wedge \star \in \mathcal{P}(S \times S \times S) \wedge \\
\forall x, y, z \in S(x \star(y \star z)=(x \star y) \star z))
\end{aligned}
$$

This predicate has infinitely many structures $X=S$, as models, as is well known (for instance, the set of real numbers endowed with addition, the set of $2 \times 2$ matrices endowed with the addition of matrices, the set of continuous real functions endowed with the composition of functions, etc.). This is a quite trivial case, but for most theories of empirical sciences, the structures are sets in a set theory like the Zermelo-Fraenkel (ZF) system (perhaps encompassing the Axiom of Choice, ZFC). The theories $T$ of our scheme (1) can also be presented in different ways, depending on the language, primitive concepts, axioms, etc. we have employed. But, fixing a particular $T$, its models are the abstract models of our scheme (the ' $A$ ' part of it), in the sense that a structure $X=(S, \star)$ obeying the above predicate is a semi-group. Thus, we see that the construction of a theory, so as the consideration of its models, depend on a meta-theory where the construction is performed, for we need to consider its tools in order to define the relevant structures. For instance, standard quantum mechanics needs unbounded operators. But in the so called Solovay set theoretical model, all operators are bounded [20]; so, how to construct a quantum mechanics within such a set theory? Suppes did not consider this point, for he works within an informal set theory in the corresponding mathematics which turns out to be ZFC. But for a deeper philosophical consideration, it is clear that we cannot leave this point behind. And this will happen mainly when we consider the possibility of alternative systems of logic and set theories in which these predicates may be developed. 
The final stage in our schema consists in the abstract models $A$. Here, ideally we have an axiomatized theory, with explicit underlying logic and axioms. It is at this step that the relation with ontology, as we intend to discuss it, becomes clearer. A system of logic indirectly involves substantial commitments that, once the logic is assumed, are introduced in any scientific theory that is grounded in such a logic (see for instance [4], [5]). Notice that the underlying logic involves several fundamental assumptions that may influence a theory: for instance if some version of the usual logical laws hold (excluded middle, non-contradiction, the law of identity), so as the question whether the logic is strong enough to ground the mathematics required for the theory, or how many truth values are allowed in the system, and so on. Obviously, all of those are relevant factors for the foundational counterpart of the theory. For instance, some systems of logic are just too weak to be the underlying system of part of classical mathematics, so they are ruled out as non-starters.

Here we shall not turn to the full discussion these issues require, but once we have arrived on the distinctions among the different stages of the elaboration of a scientific theory, we wish to consider the mutual influence between logic and ontology. We begin with what we call 'classical ontology'. This was formed from our informal view about the objects of our surroundings; the standard assumptions we make about them, in a certain sense, have shaped classical logic, standard mathematics and even classical mechanics. So, we arrive to an in re approach to scientific theories: we suppose to have some 'reality' to start with, and elaborate our scientific claims with the aim of obtaining knowledge about this reality. But, in doing that, we create something new, a 'theory' which gains a kind of autonomy and can be applied to other domains as well. Furthermore, the developments given inside the theory may suggest that the world might not be as expected, presenting some new and unsuspected details not perceived before. Physics is perhaps the best space for examples, for instance of those particles whose existence was proven 'mathematically' and only after this they were 'discovered' to exists (for instance, the celebrated case of the omega-minus particle - the reader may take a look at the October 1964 issue of Scientific American).

So, there is a twofold way: we may start with the 'reality' (either $R$ or $E R$ in our scheme), that is, by a certain ontological view, and look for the corresponding theories aiming at describing it, or we can assume a certain $I T$ or $T$ as already given in some way that look behind for the ontology (or ontologies) that can be compatible with such theories. This interplay of course has logical and ontological interests. But instead of discussing in in abstracto, let us sketch a view we term 'classical ontology'. 


\section{3. 'Classical ontology'}

In order to be applied to the real world, classical mechanics, as a mathematical theory, usually makes many idealizations and abstractions. For instance, in Newtonian mechanics we assume that the planets orbit the sun in elliptical orbits, but this is an idealization which could be true only if there were just two bodies. On what concerns logic, it is usually assumed that standard objects of our surroundings obey the laws of classical logic (at least with a good approximation): every object is identical to itself (numerical identity), for any object $o$ and any property $P$, the law of excluded middle holds, so that the object either has the property $(P o)$ or does not have it (it is not the case that $P o$ ), and no object can both have and not have the same property in the same circumstances (law of non-contradiction). Similar remarks could be made for other laws of classical logic.

Classical logic, standard mathematics and, in a certain sense, classical mechanics, are grounded on our experiences with standard medium sized objects. We call the result of this particular way to categorize the world 'classical ontology'. Of course, assuming that the world fits this description is a non-empirical hypothesis. Furthermore, the claim should not be assumed as encompassing the supposition that it is a closed 'world view'; there is plenty of space for discussion on fine details about how to articulate the view, but the general outlines are given by the constraints imposed by those theories.

To illustrate this last claim, consider the specific case of classical logic. Can we simply search for the kind of world we would have from the point of view of logic? Of course we can; so, by assuming classical logic, what are we committed with? We need to take care in this point; a straightforward answer is not available. It would not be wrong to assume that once classical logic or classical mechanics (supposed grounded on such a logic) is assumed to apply to a certain domain, the objects of this domain necessarily are already settled in their main features, however, it is not the case that they are settled in their finer details. In fact, classical logic has also a constructive interpretation (say in terms of Paul Lorenzen's approach [17]); thus, even when seen from the perspective of classical logic, the domain might be 'constructive' in some sense. So, from the point of view of logic (or mechanics) alone, we cannot decide, roughly speaking, whether the world is somehow 'constructive or not. Anyway, as we mentioned, some of general guidelines are present.

Given the combination of classical logic, classical mathematics, and classical mechanics, we may try to schematize a way of thinking by presenting some characteristics of what we call $O(C)$, for 'classical ontology', partially inspired by Peter Mittelstaedt ([21]), part by Redhead and Teller ([26]), and 
part by our own considerations. $O(C)$, which we can say maps part of the presuppositions assumed even implicitly in the formation of classical logic, may be roughly described as encompassing at least the following features:

(1) Physical objects, according to this view, are continuants, in the sense that a physical object at one time can (at least in principle) be re-identified as the same one that exists at an earlier (and at a later) time, that is, they have genidentity. Of course this feature also presupposes a lot more: $O(C)$ is partially grounded on what we call Newtonian's absolute concept of space and time and on a form of determinism, typical of classical physics. Re-identification, for all purposes, may always be effected by following an in principle complete deterministic trajectory, which every object is endowed with (with the proviso that there are no collisions). But of course we might also enable the consideration of relativistic mechanics, whose space-time is not Newtonian. Even so this characteristic of objects do not change: it is a presupposition that they continue to be themselves after modifications of their physical states.

(2) Physical objects are objects of predication; that is, they bear properties, such as position, velocity, and energy. More than that, they obey what may be called a Complete Determination Principle: we can (in principle) ascribe simultaneous values to any set of physical properties for a given object.

This point makes a link of $O(C)$ with some of the most well-known laws of classical logic. Indeed, this conception of objects is grounded on the idea that objects always either have or fail to have a given property, that is, once there are characteristics that can be attributed to them, they either instantiate those features or do not instantiate those features, and no third possibility is available (a version of the law of the excluded middle). Another basic presupposition is that an object cannot both have and not have a certain property at the same time (and this commits us with the validity of a version of the law of non-contradiction).

(3) Physical objects are individuals, in the sense that they obey some principle of individuality; that is, we may assume that there is 'something' which confers them individuality. This means that a certain object is distinct from any other object. Saying in other words, in a way that will serve to us us later, given two objects whatever, they are different and, being two, there is at least one 'feature', in general a property, that distinguishes them. The search for a Principle of Individuation has a long history in Western philosophy. The two most wellknown schools are those centered either in endorsing a form of substratum theory or that there is nothing beyond the properties of the individuals, termed bundle theories [18, Chap.3]. Both present problems on what concerns physics (for an account involving quantum mechanics, see [9]). We shall come back to this point soon. 
In classical particle mechanics, where there are no deformations, every object has a 'history' ascribed to it by the laws of motion. We can (in principle) trace the trajectory of the object and follow it in time both forwards and backwards. As Schrödinger has said a long time ago,

"[f]rom our experiences on a large scale, from our notion of geometry and mechanics - particularly the mechanics of the celestial bodies - physicists had distilled the one clear-cut demand that a truly clear and complete description of any physical happening has to fulfill: it ought to inform you precisely of what happens at any point in space at any moment of time... We may call this demand the postulate of continuity of description." [29, p.26]

The problem in saying things like that without restriction is that it is troublesome when it comes to chaotic systems. Classical systems may be chaotic, and in this case there will be no way to preview the final trajectory of a certain system. But anyway, even in continuum mechanics (with deformations caused by forces) we may assume that the trajectory does exist and that our ignorance is confined to the epistemological level, and does not infect ontology. In this sense, there is always, at least in principle, the possibility to attribute an individuating feature to any object: its trajectory.

(4) The objects in $O(C)$ may be physically indiscernible. Thus, to keep $O(C)$ intact (that is, to keep the objects' individuality), we may need to assume, as usual, that there exists something more in an object than the sum of its properties. Heinz Post said that such entities present a transcendental individuality [24]. This view, obviously, is an alternative to the individuation by the trajectory, commented on above. For instance, consider the situation below, where we have $N$ physically indiscernible objects to be accommodated into $P$ containers.

The number of possibilities can be determined; for instance, suppose $N=3$ and $P=2$, and let us illustrate the situation with a rough schema; call the containers $A$ and $B$ and the systems $a, b, c$. Thus, we have the possibilities presented in the Figure below:

\begin{tabular}{|c|c|c|}
\hline & A & B \\
\hline 1 & A,B,C & \\
2 & & A,B,C \\
3 & A,B & C \\
4 & A,C & B \\
5 & B,C & A \\
6 & A & B,C \\
7 & B & A,C \\
8 & C & A,B \\
\hline
\end{tabular}


To other cases, we can calculate the possibilities by a formula (which will not concern us here - but see [11, cap.5]). This kind of counting is called Maxwell- Boltzmann 'statistics'. Important to remark that states 3, 4 and 5 (so as $6,7,8)$ are taken as distinct because the objects are considered as individuals, as the objects of $O(C)$ are.

(5) 'Classical objects' may even be supposed to be in principle discernible from each other. Any impossibility of distinguishing them then is regarded as an epistemological ignorance only. This enables us to postulate that in having a collection of them, we can regard it as a set in some standard set theory (a collection of distinct objects, according to Cantor's well known 'definition' [3, p.85]). And, having a finite number of objects in $O(C)$, we can attribute a cardinal number to the collection. In this sense, this hypothesis says that there cannot be solo numero indiscernible objects. So, if our ontology will admit this possibility (as we shall suggest below), then standard set theories can be questioned in expressing them (we beg your patience for now). This does not prevent that indiscernible objects may be treated in such a framework, but this will require some mathematical tricks in order to cope with indiscernibility, a price we must pay if we intend to keep classical logic intact. The easiest way to do that is to follow Hermann Weyl's strategy of assuming a set $S$ (say, with cardinality $n$ ) and an equivalence relation $\sim$ defined over $S$. Thus, the equivalence classes of the elements of $S$ by the relation $\sim$ play the role of classes of indiscernible objects [32, App.B]. But in doing that we have forgotten that the objects were taken from the beginning as discernible elements of the set, so something seems to be wrong with the formalism that intends to capture indiscernibility (for a discussion, see [9]). We shall return to this point below.

\section{A possible ontology for quantum mechanics}

As we have said before, physical theories do not determine a priori and univocally their ontologies. The issue gets even more complicated when we notice that even if a physical theory has an intended interpretation, in general it can be later applied to other domains, dealing with very different kinds of things (consider applications of the mathematical apparatus of quantum mechanics to economy). Particular theories may have a specific behavior, but in the general setting, the possibility of alternative interpretations are not ruled out a priori. As is well-known, the formalism of non-relativistic quantum mechanics (we leave quantum field theories out of this discussion) is compatible with a plurality of incompatible ontologies.

Now, to narrow the field and advance in the search for one specific 
ontology, let us first describe, in analogy with what we have exposed before, a minimum nucleus of a possible 'quantum ontology'. We shall term it $O(Q)$, and we beg the reader to understand that we are not claiming that this ontology is the only one compatible with quantum mechanics. We are just describing what could be, roughly, an ontology for quantum mechanics in which identity and individuality are placed at the center of the concerns.

(1) In the 'classical' setting (compatible with $O(C)$ ), it is assumed that all objects can be re-identified through time. There is an old problem concerning what makes this re-identification possible, but it is a standard assumption (we could say that it is a postulate) that they can be re-identified. Quantum objects, in most interpretations, and here also in our $O(Q)$, lack this characteristics in certain situations as when their wave functions overlap. Thus, we are endorsing the failure of Schrödinger's above mentioned principle of continuity of description (see page 9); as he has put it, "[i]t is this postulate of continuity that appears [in quantum mechanics] to be unfulfillable!" (loc.cit.). For the sake of completeness of the description, of course we should consider Bohm's quantum mechanics, which postulates that the objects' trajectories never overlap. As we mentioned, there is not a single way to sort out the main features of quantum objects, but we shall leave Bohmian theory aside for now, given that it introduces further intricacies and falls on the "hidden variables" approach.

(2) By 'to have identity' we may understand the following: the object has something (the nature of this 'something' given either by substratum theories or by bundle theories of individuation) that makes it an individual. Let us remark that even though objects in $O(C)$ may be taken as indiscernible from other ones in some situations, as we have said before, this indiscernibility does not pose a threat to their individuality, for they may be individuated by some transcendental identity or even by their trajectories. Now, the situation is different in quantum mechanics. The objects of $O(Q)$ (in some situations) cannot be discerned from similar ones, and there is no reason to suppose that they are individuated by transcendental individuality.

In order to see this point from another perspective, suppose we have two quantum objects, say two electrons, one here and another in Mars. They can be described by distinct wave-functions $\psi 1$ and $\phi 2$ and hence they can be identified as Paul and Peter. But the problem appears when we try to describe the joint system, for then we need to use an anti-symmetric wave-function (in the case of electrons), and in this case we shall have a linear combination of the two wave-functions without being able to tell who is Peter and who is 
Paul. We are able to go as far as to know that we have two entities, one here and another there, but cannot say which is which, there is no need to introduce a transcendental principle of individuality. This is a typical characteristic of quantum objects according to the standards, and is assumed in $O(Q)$.

Suppose we have a neutral Helium atom. As it is known, it has two electrons in a superposed anti-symmetric state. It is possible to ionize the atom by releasing one of the two electrons, getting a negative ion. It is clear that in this process one electron was released. Now we can obtain a neutral atom again by making the ion to absorb an electron. A chemical fact is that the 'new' neutral atom will have all the same properties (values of any observable) of the 'old' one. Everything that can be inferred from one of them can be inferred also from the other as well. The question is: is the new neutral atom the same as the neutral atom ionized before? In the same vein, we can ask: is the released electron the same as the absorbed electron? Apparently, these questions cannot be answered non-arbitrarily.

From the physical or chemical points of view, this kind of question does not even matter. As we have said, the results taken from the first atom and from the second one are exactly the same, and that is all. Let us exemplify once more. In the combustion of methane, escrever $\mathrm{CH} 4+2\left(\mathrm{O}_{2}\right) \rightarrow \mathrm{CO} 2+2(\mathrm{H} 2 \mathrm{O})$, one methane molecule reacts with two Oxygen molecules to produce carbon dioxide and water vapor. As we see, from the four Oxygen atoms present in the two Oxygen molecules, two go to the dioxide, and two go to the water vapor. But, which ones? It does not matter. Chemistry does not depend on this assumption. The individuality of these objects is not something required to the theory. The only important thing is the nature of the objects and the number of them.

Based on examples like this one, we assume that, according to $O(Q)$, the objects do not present identity conditions, in the sense that there is no a principle of individuation that enables us to ascribe to one of them an identity that may serve to re-identify it in a later situation, say by identifying which particular Oxygen atoms are now forming the carbon dioxide.

(3) In classical physics, one of the basic assumptions is that given a certain collection of properties of an object, all these properties can have definite values simultaneously. This is called the Value Definiteness Problem. The difficulty in effectively finding these values is of another nature, but it is an assumption typical of $O(C)$ that they exist and are well determined. In the quantum domain, there are restrictions to this principle. It is in fact one of the typical characteristics of quantum mechanics that we cannot ascribe definite values to all sets of observables at once. There are no-go theorems say- 
ing precisely that; perhaps the most important one being the Kochen-Specker theorem (see [25]).

(4) Quantum objects may enter in states of superposition. This is perhaps the great novelty of quantum physics. A particular case is that of entanglement, which Schödinger regarded as being the characteristic trait of quantum mechanics, and today quite important for new developments in physics, such as quantum teleportation and quantum information (the interested reader will find in [2] useful information). This is in particular the case of the two electrons of the neutral Helium atom mentioned above. They are entangled. So, although (in a given direction) they will present (when measured) the values of their spin with opposite values (say, $1 / 2$ and -1/2), it is impossible to say which electron has which particular value.

(5) Given the above, we cannot regard the objects of $O(Q)$ as individuals in the sense of $O(C)$. Taking a terminology (perhaps a bad one) from the beginnings of quantum mechanics, we say that the objects in $O(Q)$ are non-individuals. Heisenberg, Schödinger and others have said that, due to the fact that in quantum physics objects like elementary particles could no more be identified, that they had lost their individuality. According to $O(Q)$, there is nothing to lose, for they do not have individuality from the start. This is precisely the main objective of introducing $O(Q)$ : to pursue such an ontology right from the start (as suggested by Heinz Post [24]). Important to say that this assumption does not avoid that a quantum object may be isolated. This is done every day in physics. But the important difference to the objects of $O(C)$ is that the properties of a certain isolated quantum object are exactly the same as of 'another' quantum object of the same kind, whatever it is. And this in blatant distinction from $O(C)$.

Another typical aspect of quantum objects concerns their behavior regarding possible combinations. Let us suppose a situation such as that one described in $O(C)$ at page 13. Three quantum objects of the same kind are to be distributed into two containers. Assume further that they are indiscernible bosons, so that they share the same state (container). Then the possibilities are given by the table below. There is also here a way of counting how many situations there are (see [11, chap.5]). (The figure below is just an heuristic device; there is no sense in representing quantum objects by little balls): ${ }^{5}$

5 Planck's formula reads $(N+P-1) !$, where $N$ is the number of particles and $P$ is the number of possible states. $\quad N !(N-P)$ ! 


\begin{tabular}{|c|c|c|}
\hline & A & B \\
\hline 1 & $\bullet$ & \\
2 & & $\bullet$ \\
3 & $\bullet$ & $\bullet$ \\
4 & $\bullet$ & $\bullet$ \\
\hline
\end{tabular}

Now, we cannot even call $a$ one of them; which one would be $a$ ? The objects are now indiscernible in such a way that we cannot represent them as different, even if the difference comes as attributed through a label. If they are fermions, then they cannot share the same quantum state, and the only possibility would be one of them in $A$ and another one in $B$, but no distinction is possible if we permute one of them by the other that remained out. The first way of counting (for bosons) is called Bose-Einstein 'statistics', and the later one the Fermi-Dirac 'statistics'.

The reader may be thinking that we are strongly committed to the idea that quantum objects can be viewed as little balls. We have emphasized that this is not so. Thus, in order to show a typical situation in quantum field theory, let us show you a figure taken from Wolfgang Ketterle's Nobel Lecture [12]. The figure shows some situations involving quantum objects by relating their wavelengths and the temperature. It is firstly shown that these quantities are inverse one each other. Then, as the temperature $T$ decreases, the wavelengths become longer, until $T$ becomes near the absolute zero (just few pico-Kelvins). In this case, the waves become so long that all of them are seen as acting as if they were just one big wave. Then we have a BEC, a Bose-Einstein Condensate, and no distinction is possible: the bosons are in the same quantum state, and so have all their characteristics shared among them.

Of course bosons need to be dealt with within QFT (quantum field theories), but our discussion on quantum objects do not need to be restricted to non-relativistic quantum mechanics. But QFT poses another difficulty we shall only mention here. Most of them are local theories. This intuitively means that the quantities that characterize a physical field are given at all points in space-time, and the interaction of the fields is determined by their values at the coincident points of space-time, that is, and this is referred to as they being local. The problem is that this leads to the appearance of divergences which are physically meaningless, that is, infinitely large values for some physical quantities. Then nonlocal quantum field theories have been proposed to overcome this difficulty with divergences. So, yet one could say that being local, the position in space-time could serve as a principle of individuation, the same problem posed before remains: due to the intrinsic characteristics of quantum objects (even in QFT), any permutation leads to the same results. Non-individuality is present also here. 


\section{Looking for a mathematical framework}

According to the interpretation that gives rise to $O(Q)$, quantum objects may be indiscernible in a way that there is no physical means to discern them. But, is there another option? Recall that classical objects, that is, those in $O(C)$, may also be indiscernible this way. But in this case they are discernible by a principle encoded in $O(C)$. The mathematical framework we use to express $O(C)$, namely standard set theory (of course this is just one of the options), as we have seen, is compatible with the idea: collections of classical objects may form sets, these objects obey classical logic, and every difficulty concerning their possible distinguishability is regarded as an epistemological issue.

As we have suggested before, the same mathematical framework (encompassing classical logic), namely a standard set theory, can be used to ground

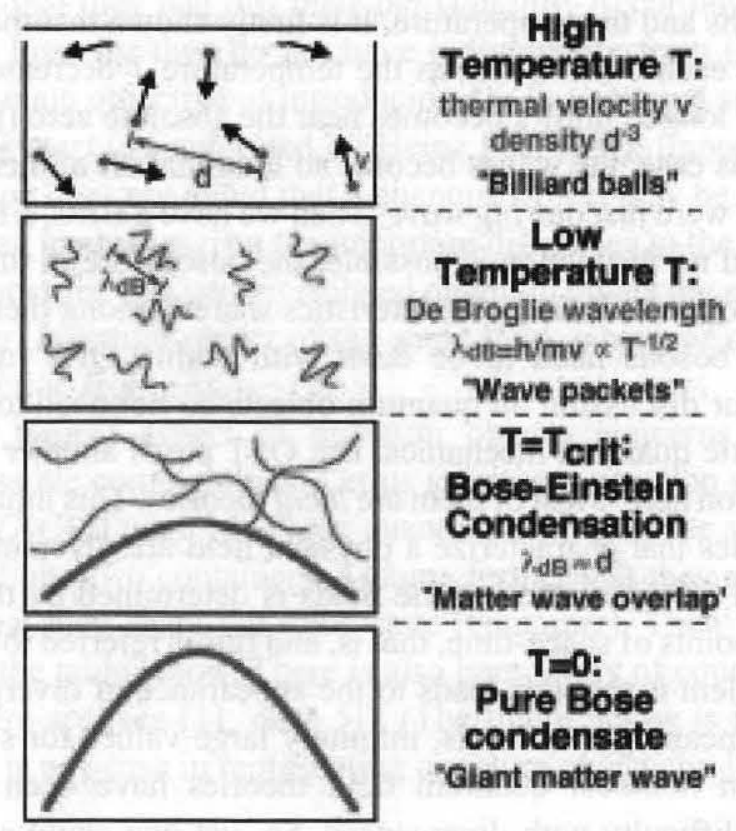

Figure 1: Bose-Einstein Condensate, BEC. Qc The Nobel Foundation. 
the typical quantum formalism, but at the expense of some adjustments, such as Weyl's strategy. In other words, we can proceed by choosing a suitable congruence relation to cope with indiscernibility. But, we know, at the bottom the objects are assumed to be discernible; we are just pretending that they are not. The usual procedure goes more or less as follows.

We start by labeling the objects as if they were distinguishable, say $a$ and $b$ for the two electrons of an Helium atom to use once more our example mentioned before. Then we demand that all physical measurable meaningful results must be independent of this label attribution; for instance, in our example, we write $|\uparrow a\rangle$ to say that object $a$ has spin up in a given direction, and $|\downarrow a\rangle$ to say that it has spin down in that direction. Thus, in order to say that one of them has spin up in the direction while the other one has spin down, a state termed $|\phi\rangle$, we write

$$
|\psi\rangle=(1 / \sqrt{2})\left(\left|\uparrow_{a}\right\rangle \cdot\left|\downarrow_{b}\right\rangle-\left|\uparrow_{b}\right\rangle \cdot\left|\downarrow_{a}\right\rangle\right)
$$

the dots stand for the tensor product and $1 / \sqrt{2}$ is a normalization factor. An exchange of labels leads to the vector $-|\psi\rangle$, but this does not matter; what is relevant is $|\psi|^{2}$, which gives us the probabilities, so the labels do not represent nothing relevant here. So, no exchange of labels (which indicates a permutation of particles) is physically detected. But we have detected it! And so mathematics does! Some philosophers have claimed that we should use the Fock space formalism instead, for in this case we would be free of the labels (see [26]). But the trick remains due to the underling mathematics, which continues discerning them (as pointed out in [9, chap.9]).

An ontology compatible with $O(Q)$ should not use such tricks. Recalling Post once more, the indiscernibility of the entities should be pursued right from the start or, as suggested by John Stachel, "from bottom to top" [30]. ${ }^{6}$ Then, in order to cope with $O(Q)$, we are in need of a different mathematical and logical basis. We do not intend to present this mathematics here, but just mention that it can be seen in [9], and with respect the way to express the quantum formalism without presupposing labels, in [8].

${ }^{6}$ Using his terminology, we can say that the loss of individuality made by suppositions like Weyl's strategy are "top down", while the assumption of non-individuals from the start would be "bottom up" 


\section{Discussion}

As we have argued, mathematical theories and set theory in general encompass some of the assumptions of $O(C)$ given that they help to shape $O(C)$ itself. However, those theories are powerful enough in order to develop quantum theory. Now, to keep in touch with the consequences of such a theory in the ontological side, at least, it seems one is advised to look for a more appropriate system of logic, one that allows us to codify those features shown by quantum objects as described above. So, in this sense, the theory influences from the top the underlying logic, that is, there is a top-bottom influence. Once we come up with such a logic and set theory, we are able to investigate how they influence the theory from the bottom; perhaps, such a logic may enlighten some part of the theory.

In the case of quantum mechanics, in particular concerning identity and indiscernibility, one such system was indeed proposed: quasi-set theory (see French and Krause [9]). Quasi-set theory encapsulates the idea that identity and indiscernibility are distinct relations, and makes it explicit in the system. Also, it does separate concepts of counting and cardinality. So, even if we begin by using classical logic and mathematics to develop quantum theory, when it comes to metaphysical discussion one can develop distinct systems of logic that reflect more closely the features the relevant objects have.

By failing to notice the incompatibility, or by assuming that, for instance, quantum objects may really be indiscernible, while at the same time keeping classical logic, one is somehow re-introducing the very concepts the theory (under the interpretation we proposed by describing $O(Q)$, of course) seemed to dismiss. In this sense, classical logic and mathematics in general may be seen as introducing metaphysical assumptions through the backdoor.

In this sense, for those willing to keep classical logic and mathematics intact, there is the alternative of adjusting other parts of the whole theory to accommodate the strange features of quantum mechanics. For instance, one must look for explanations on quantum statistics and quantum indiscernibility that may be compatible with quantum phenomena. In this sense, the 'corrections' are made by re-interpreting phenomena at the top, on the theory itself, and leaving the 'bottom', logic and mathematics, with no revisions. Both proposals are possible, but we have suggested that a revision of logic and set theory itself in the light of quantum phenomena may be a welcome addition to our understanding of those entities.

To contrast the proposal offered in this work, which sought to show how one may end up revising the logic by judging from a particular ontological interpretation of the results of the theory $(\mathrm{QM})$, we may present briefly a pro- 
posal advanced by Saunders (in [28]), comparing it with our proposal. According to Saunders, roughly speaking, it is not clear which kind of objects quantum mechanics deals with. In order to make that point clearer, one is advised to keep classical logic as an auxiliary tool and just check whether there are means to present formulas in a first-order regimented language of quantum mechanics which could help us discern quantum objects. As is already well-known, Saunders concluded that fermions may be weakly discerned, i.e., discerned by a symmetric and irreflexive relation, while bosons are not even weakly discernible, so that, at least in that work, they were simply not deemed to be objects by Saunders' lights. Of course, Saunders agrees that quantum entities are not discernible by properties; his claim is, however, that for fermions there is always a relation discerning those entities. This move was meant to show that a version of the Principle of the Identity of Indiscernibles (PII) is valid in quantum mechanics; while fermions do obey it, bosons are not a counterexample, their failure to satisfy the principle means that they are not legitimate objects.

This approach has many interesting aspects we are ignoring here because of the lack of space. Our main point is to call the reader's attention to the fact that first-order classical logic along with the PII plays the role of a 'tailoring tool', so that together with the weak discernibility relations provided by quantum mechanics one has a method for providing for the entities of the theory. Furthermore, these entities are classical objects in the traditional sense (or so Saunders claims).

Notice that there is a clear difference with our approach. We keep quantum mechanical information about the nature of the objects and look for a more adequately developed logic which encompasses the features of those objects. The plan is to build in the logic itself those features which seem to be required on the objects by the theory, at least according to a sensible interpretation in which they are not individuals (sensible to our mind, anyway). Saunders' strategy reverses the procedure: we keep logic intact and judge, with the help of this very logic and a first-order version of PII, which among the posits of quantum mechanics do satisfy the classical Quinean requirement for an entity to a legitimate object in such a logic: it must have identity conditions. By being weakly discernible, it seems, fermions fit the request, bosons do not, and are accounted for in terms of modes of a quantum field.

We believe that Saunders' proposal, ingenious as it is, falls prey to the problem we have already mentioned: inside the classical mathematical framework, there is always means to distinguish things, the mathematics is just too strong. As we have argued, there are no indiscernible objects. In fact, more than that is true, inside classical set theory one may always distinguish objects 
by a property, not merely by a weakly discerning relation, as Saunders requires. In this sense, an absolute kind of discernibility enters through the backdoor by the adoption of classical mathematics. We believe that our approach is not prey to such objections, given that it was made to account precisely for those features of quantum particles.

It seems to us that logic need not be kept sacrosanct in the discussion of ontology. As we have mentioned, Saunders' position is interesting and legitimate, but we feel it presents some problems. By adjusting the logic also to account for the entities a theory deals with one brings logic itself to the center of the dispute, allowing for a more interesting investigation in search for a kind of reflexive equilibrium between the demands of logic and the demands of a specific theory. This would do more justice to the idea that science as a whole is involved in the investigation of the nature of reality: that includes logic itself. So, in this sense, logic is not immune to revision, and we include it seriously among those of our tools used for the investigation of reality (but this is an issue we have no space to discuss here).

Of course, we are aware that this kind of move proposed here does not solve once and for all the problems associated with the ontology of quantum mechanics. However, it gives us freedom to investigate how logic could be like were the strictures of quantum mechanics to hold for every object. As it was shown in [8], a completely coherent version of quantum mechanics may be erected on the basis of this logico-ontological investigations. In this case, one may be sure that indiscernibility is built-up from bottom up, making logic compatible with the strictures of the theory. The road is open for us to investigate how this move can shed some light on quantum mechanics and its ontology!

\section{References}

[1] Browder, F. E. [1976], Mathematical Developments Arising from Hilbert Problems (Proceedings of Symposia in Pure Mathematics, Vol. 28, Part.1). Providence: American Mathematical Society.

[2] Bub, J. [2014], 'Quantum Entanglement and Information', The Stanford Encyclopedia of Philosophy (http://plato.stanford.edu/archives/fall2014/entries/ qt-entangle/).

[3] Cantor. G. [1955], Contribution to the Founding of the Theory of Transfinite Numbers. New York: Dover.

[4] da Costa, N. C. A. [1980], Ensaio sobre os Fundamentos da Lógica. São Paulo: Hucitec.

[5] da Costa, N. C. A. [2002], Logic and ontology, Principia 6 (2), 279-98.

[6] Dewey, J. [1938], Experience and Education. Toronto: Collier-MacMillan Canada Ltd. 
[7] d'Espagnat, B. [1983], In Search of Reality. New York: Springer.

[8] Domenech, G., Holik, F. and Krause, D., 'Q-spaces and the foundations of quantum mechanics', Foundations of Physics 38 (11), 969-994

[9] French, S. and Krause, D., [2006], Identity in Physics: a Historical, Philosophical and Formal Analysis, Oxford: Oxford Un. Press.

[10] Galilei, G. [1967], Dialogue Concerning the Two Chief World Systems: Ptolemaic and Copernican. New York: The Modern Library.

[11] Griffiths, D. J. [2005], Introduction to Quantum Mechanics, 2nd.ed. New Jersey: Pearson Prentice Hall.

[12] Ketterle, W. [2001], Nobel Lecture. The Official Website of the Nobel Prize: http:// www.nobelprize.org/nobel prizes/physics/ laureates/2001/ketterle-lecture.html

[13] Husserl, E. [1970], The Crisis of European Sciences and Transcendental Phenomenology. Trans. David Carr. Evanston: Northwestern Un. Press.

[14] Kosso, P. [1998], Appearance and Reality: An Introduction to the Philosophy of Physics. New York: Oxford Un. Press.

[15] Krause, D. and Arenhart, J. R. B. [2014] Logical reflections on the semantic approach. Forthcoming.

[16] Krause, D.; Arenhart, J. R. B.; Moraes, F. T. F., [2011] Axiomatization and models of scientific theories. Foundations of Science, 16, 363-382.

[17] Lorenzen, P. [1971], Differential and integral: a constructive introduction to classical analysis. Un. Texas Press.

[18] Loux, M. J. [1998] Metaphysics: a contemporary introduction. Routledge: London and New York.

[19] Magalhães, J. C. and Krause, D. [2001], Suppes predicate for genetics and natural selection, Journal of Theoretical Biology 209 (2), 141-53.

[20] Maitland Right, J. D. [1973], All operators on a Hilbert space are bound. Bull. Am. Math. Soc. 71 (6), 1247-1250.

[21] Mittelstaedt, P., [2005], 'Quantum physics and classical physics - in the light of quantum logic'. International Journal of Theoretical Physics 44(7), 771-781.

[22] Nagel, E., Suppes, P. and Tarski, A. (eds.) [1962], Logic, Methodology and Philosophy of Science: Proceedings of the 1960 International Congress. Stanford: Stanford Un. Press.

[23] Penrose, R. [2004], The Road to Reality. New York: Vintage Books.

[24] Post, H. [1963] Individuality in physics. The Listener, 10 October 1963, 534-537. Reprinted in (1973) Vedanta for East and West, 14-22.

[25] Redhead, M. [1987], Incompleteness, Non-Locality and Realism: A Prolegomenon to the Philosophy of Quantum Mechanics. Oxford: Clarendon Press.

[26] Redhead, M. and Teller, P., [1991], 'Particles, particle labels, and quanta: the toll of unacknowledged metaphysics', Foundations of Physics 21, 43-62.

[27] Rovelli, C. [2013], Aristotle's physics. <arXiv:1312.4057v1>

[28] Saunders, S. [2003] Physics and Leibniz's Principles. In: Brading, K., and Castellani, E., (eds), Symmetries in Physics: Philosophical Reflections, Cambridge:Cambridge Un. Press, 289-307.

[29] Schrödinger, E. [1952], Science and Humanism. Cambridge: Cambridge Un. Press.

[30] Stachel, J. [2005], 'Structural realism and contextual individuality', in Ben- Menahem, Y. (ed.), Hilary Putnam. Cambridge: Cambridge Un. Press, 203-219.

[31] Suppes, P. [2002], Representation and Invariance of Scientific Structures. Stanford: CLSI.

[32] Weyl, H. [1949], Philosophy of Mathematics and Natural Science. Princeton: Princeton Un. Press. 


\begin{abstract}
In this basically expository paper we discuss the role of logic and mathematics in researches concerning the ontology of scientific theories, and we consider the particular case of quantum mechanics. We argue that systems of logic in general, and classical logic in particular, may contribute substantially with the ontology of any theory that has this logic in its base. In the case of quantum mechanics, however, from the point of view of philosophical discussions concerning identity and individuality, those contributions may not be welcome for a specific interpretation, and an alternative system of logic perhaps could be used instead of a classical system. In this sense, we argue that the logic and ontology of a scientific theory may be seen as mutually influencing each other. On the one hand, logic contributes to shape the general features of the ontology of a theory; on the other hand, the theory also puts constraints on the possible understanding of ontology and, respectively, on possible systems of logic that may be the underlying logic of the theory.
\end{abstract}

Keywords: logic; classical ontology; quantum ontology; quantum mechanics; identity. 